\title{
Current practice trends of oedema management in the hands of people with tetraplegia in Australia
}

\author{
Soo $\mathrm{Oh}^{1,2} \cdot$ Louise Gustafsson ${ }^{1,3} \cdot$ Sally Eames ${ }^{1,4}$ \\ Received: 28 February 2019 / Revised: 5 June 2019 / Accepted: 18 July 2019 \\ (c) The Author(s), under exclusive licence to International Spinal Cord Society 2019
}

\begin{abstract}
Study design Survey research design.

Objectives To describe current practice methods for oedema management in people with tetraplegia.

Setting Australia.

Methods Online survey with open and closed questions regarding clinical practice trends in the assessment and treatment of oedema in the hands in people with tetraplegia.

Results Seventeen occupational therapists working in spinal cord injury (SCI) in Australia completed the survey. Oedema was identified by visual inspection $(n=17,100 \%)$ and recorded using circumferential tape measurement $(n=13,76 \%)$. Elevation was used by all participants in conjunction with compression gloves $(n=13,76 \%)$, retrograde massage $(n=13$, $76 \%)$, compression bandaging $(n=12,71 \%)$ and the boxing glove splint $(n=9,53 \%)$. Participants stated that oedema presented challenges to patients with difficulty exercising $(n=11,65 \%)$, changes to body image $(n=5,29 \%)$ and pain $(n=$ $4,24 \%)$.

Conclusion Assessment and treatment practices were not consistent. Oedema in the hands in people with tetraplegia was perceived to have various impacts on a person's rehabilitation and hand function. The findings highlight the need for research evidence to guide practice.
\end{abstract}

\section{Introduction}

People who sustain tetraplegia following a spinal cord injury (SCI) report that hand and arm function is the most important factor in improving their quality of life [1]. The loss of function to their upper limbs can be a key link to their level of mobility, independence and engagement with occupational roles. A range of secondary complications such as contractures, pain and oedema in the hands can have significant implications to hand function. Key SCI clinical practice

Supplementary information The online version of this article (https:// doi.org/10.1038/s41394-019-0215-7) contains supplementary material, which is available to authorised users.

Soo Oh

soo.oh@health.qld.gov.au

1 School of Health and Rehabilitation Sciences, University of Queensland, Brisbane, QLD, Australia

2 Occupational Therapy department, Princess Alexandra Hospital, Brisbane, QLD, Australia guidelines highlights the importance of preventing secondary complications in the upper extremity including oedema [2].

Oedema is defined as the accumulation of fluid in the intracellular spaces due to an imbalance of effective capillary pressure or an obstruction to the venous lymphatic flow by a weak or paralysed extremity [3]. Oedema is well researched in areas such as stroke and lymphoedema and has been linked to reduced range of motion (ROM), pain and compromised hand function [4, 5]. However, there has been minimal research conducted specific to cause, incidence or impact of oedema after SCI. With limited evidence to support assessment and treatment practices, clinicians working in SCI often refer to evidence from other areas.

3 School of Allied Health Sciences, Griffith University, Brisbane, QLD, Australia

4 Community and Oral Health, Metro North Hospital and Health Service, Brisbane, QLD, Australia 


\section{Assessment tools}

The International Spinal Cord Injury Upper Extremity Basic Data Sets (ISCIUEBDS) states that oedema can be graded as 'minor' or 'major' and that impact on function can be categorised as 'minimal', 'moderate' or 'extensive' [6]. However, the ISCIUEBDS make no specific recommendations regarding assessment tools. A tape measure to record the circumference of the affected limb is the assessment tool most commonly used for upper limb lymphoedema [7] followed by the volumetric metre and perometry. The volumetric metre is used to measure water displacement when the affected limb is immersed in water. A perometer is an infra-red measuring device that calculates volume of the extremity [8]. Emerging assessment tools for lymphoedema include bioimpedance spectroscopy [9] and the Moisturemeter-D [10]. The bioimpedance spectroscopy measures the resistance of body tissue to electrical current flow in the extracellular fluid and demonstrates significant clinical utility and use for the early detection of the accumulation of extracellular fluid [11]. The Moisturemeter-D measures the tissue dielectric constant by measuring the local tissue water and has demonstrated reliability, showing promise in detecting change in measuring head and neck lymphoedema [10].

\section{Intervention}

The most commonly applied and effective method for the treatment of lymphoedema is compression therapy, including bandages and garments [12]. A systematic review of stroke-specific literature found the most effective treatment methods were compression garments, bilateral passive ROM and contemporary therapies [13]. A treatment method that appears to be unique to SCI is the 'boxing glove' splint [14]. The 'boxing glove' splint involves wrapping the hand in a crepe bandage with the wrist is positioned at $45^{\circ}$ of extension, the metacarpophalangeal joints at $90^{\circ}$, interphalangeal joints at $20-30^{\circ}$ and the thumb opposed with fully stretched web space. There has been limited research of this treatment method. A final, emerging treatment is kinesio tape. Kinesio tape is an elastic stretch tape that is applied to the skin's surface with claims that by lifting the skin, there are physiological effects including 'removing congestion of lymphatic fluid under the skin' [15]. There is currently insufficient evidence to suggest that the use of the kinesio tape is more effective than other treatment modalities [16].

\section{Current study}

Oedema in the hands of people with SCI is a greatly underresearched area. The purpose of this study was to gather information from clinical experts regarding their perceptions and management of oedema, including perceived causes, prevalence, assessment, treatment and effects on rehabilitation and function.

\section{Methods}

A survey research design collected information from clinical specialists in the area of SCI.

\section{Participants}

Participants for this study included occupational therapists with experience working with people with a SCI. Invitations were sent via a SCI specific email listserv and an email to the lead occupational therapist in the SCI centres in Australia.

\section{Procedure}

The email provided background information to the study and a link to the online survey in SurveyMonkey ( $)$. The survey included an introduction and sought participant consent prior to commencing. Participants were provided with an email address to contact the research team with any queries regarding the survey.

\section{Instruments}

The survey included closed and open-ended questions and was pilot tested with two colleagues. The survey gathered demographic information including place of work and level of experience and information regarding the perceived prevalence of oedema, assessment and treatment methods used, impact on rehabilitation, and any other challenges. The survey questions were developed from the existing literature and clinical expertise.

\section{Data analysis}

Data from the online platform were downloaded into Microsoft ${ }^{\circledast}$ Office Excel for collation and analysis. The closed-ended questions and Likert scale data were summarised and presented as number and percentages. Data from open-ended questions were collated and content analysis allowed categories for responses to be developed. Direct quotes are reported where appropriate.

\section{Results}

\section{Participants}

Responses were received from 17 occupational therapists and the demographics are reported in Table 1. 
Table 1 Participant demographics

\begin{tabular}{lc}
\hline Demographic & $n(\%)$ \\
\hline Service type & \\
Spinal injuries unit & $14(82)$ \\
(NSW) & $6(35)$ \\
(VIC) & $5(29)$ \\
(WA) & $2(12)$ \\
(QLD) & $2(12)$ \\
(SA) & $2(12)$ \\
Neurorehabilitation & $2(12)$ \\
Community & $1(6)$ \\
No. of years of experience as an occupational therapist & \\
0-5 years & $4(24)$ \\
6-10 years & $6(35)$ \\
$11-20$ years & $4(24)$ \\
21-30 years & $1(6)$ \\
30+ years & $2(12)$ \\
No. of years of experience working in spinal cord injury & \\
0-5 years & $8(47)$ \\
6-10 years & $5(29)$ \\
11-20 years & $3(18)$ \\
21+ years & $1(6)$ \\
\hline &
\end{tabular}

\section{Patient demographic}

Participants treated patients within 6-weeks post injury ( $n$ $=9,53 \%)$; from 6-weeks to 3 -months post injury $(n=4$, $24 \%)$; or at a range of time points on the continuum $(n=8$, $47 \%)$. The length of stay was under 3 months $(n=5,29 \%)$; 4-12 months $(n=10,59 \%)$ and over 12 months $(n=2$, $12 \%)$. Finally, $88 \%(n=15)$ of the participants reported treating their patients one to twice a day for rehabilitation and $12 \%(n=2)$ saw patients by referral only.

\section{Causes and prevalence of oedema}

Participants identified positioning $(n=17,100 \%)$ and lack of movement $(n=16,94 \%)$ as the predominant causes of oedema. They reported that oedema occurred often $(n=11$, $65 \%)$ and was more likely to occur in both hands $(n=14$, $82 \%)$ than one hand $(n=3,18 \%)$. (Table 2) Participants reported that $<50 \%$ of their current clinical caseload had oedema in their hands and there was no clear trend to 'when' oedema was likely to occur post injury.

\section{Assessment}

Oedema was most commonly identified by visual inspection ( $n=17,100 \%)$ then followed by a combination of formal and informal assessment tools. The assessment tools used by the participants are reported in Table 3. The tape
Table 2 Cause and prevalence of oedema in hands in people with tetraplegia

\begin{tabular}{lc}
\hline Cause of oedema ${ }^{\mathrm{a}}$ & $n(\%)$ \\
\hline Positioning & $17(100)$ \\
Lack of movement & $16(94)$ \\
Disuse & $11(65)$ \\
Affected sympathetic nervous system & $5(29)$ \\
Affected lymphatic system & $4(24)$ \\
Other (co-morbidities i.e. cardiac conditions) & $1(6)$ \\
\hline General impressions of prevalence of oedema & \\
\hline Never & $0(0)$ \\
Occasionally & $5(29)$ \\
Often & $11(65)$ \\
Always & $1(6)$ \\
\hline$\%$ of prevalence of oedema in the current clinical setting & \\
\hline $0 \%$ & $5(29)$ \\
$1-25 \%$ & $2(12)$ \\
$26-50 \%$ & $5(29)$ \\
$51-75 \%$ & $2(12)$ \\
$76-100 \%$ & $0(0)$ \\
Unclear & $3(18)$ \\
\hline Timing of when oedema occurs & $2(24)$ \\
\hline First 2 weeks & $2(12)$ \\
1-2 months & $2(12)$ \\
Varies & $4(24)$ \\
Unsure & \\
\hline & \\
\hline
\end{tabular}

${ }^{\mathrm{a}}$ Multiple responses permitted

measure was reported as the most effective assessment tool $(n=10 ; 59 \%)$ and one of the most commonly used $(n=13$, $76 \%$ ), seen as 'objective' and 'easy'. ROM was commonly used ( $n=14,82 \%)$, considered 'objective' but reported less likely to be effective $(n=4 ; 24 \%)$. The participants who preferred the use of photos $(n=3 ; 18 \%)$ referred to the 'visual' nature of this approach. Appearance to the naked eye $(n=2,12 \%)$ and patient feedback $(n=1,6 \%)$ were considered least effective although they were reported as 'most convenient' and 'quick'.

\section{Re-assessment}

Re-assessment occurred 4-5 times a week $(n=10,59 \%)$, once a week $(n=5,29 \%)$, and variably on admission and discharge, each visit, rarely, or every few weeks $(n=1,6 \%$ respectively). Fourteen (82\%) participants did not specify the type of assessment with the remaining three (18\%) participants specifying that a visual inspection was used. 
Table 3 Assessment tools currently used in clinical settings

\begin{tabular}{lcc}
\hline Assessment tools $^{\mathrm{a}}$ & $\begin{array}{l}\text { Currently } \\
\text { used (\%) }\end{array}$ & $\begin{array}{l}\text { Considered } \\
\text { effective }(\%)\end{array}$ \\
\hline Naked eye & $17(100)$ & $2(12)$ \\
Range of motion & $14(82)$ & $4(24)$ \\
Tape measure & $13(76)$ & $10(59)$ \\
Patient feedback & $12(71)$ & $1(6)$ \\
Comparison with & $11(65)$ & $0(0)$ \\
other hand & $11(65)$ & $0(0)$ \\
Pitting hand & $10(59)$ & $3(18)$ \\
Photos & $2(12)$ & $0(0)$ \\
Sensation assessment & $1(6)$ & $0(0)$ \\
Ill-fitting splints & $0(0)$ & $0(0)$ \\
Moisture metre & $0(0)$ & $0(0)$ \\
CT/X-ray/US & $0(0)$ & $0(0)$ \\
Volumetric metre & $0(0)$ & $0(0)$ \\
Bioimpedence &
\end{tabular}

${ }^{a}$ Multiple responses permitted

\section{Challenges with assessment}

Participants identified that the process of assessment was not consistent ( $n=5,29 \%$ ), had poor inter-rater reliability $(n=4,24 \%)$, the measures were subjective $(n=2,12 \%)$, inaccurate $(n=2,12 \%)$ and time consuming $(n=2,12 \%)$. One participant stated that assessments do not provide a precise measurement of oedema and that "presence and reduction of oedema is easy to identify visually and through patient/family feedback'.

\section{Treatment}

Elevation was the most commonly used treatment method $(n=17,100 \%)$ and seven $(41 \%)$ participants reported it to be effective. Table 4 shows the frequency of the remaining treatment methods, including reports of perceived effectiveness. Eleven participants $(65 \%)$ stated that they would use a combination of various treatment methods including bandaging for acute oedema and compression garments for chronic oedema.

\section{Challenges with oedema management}

Participants reported they felt the treatments did not have long-term effects $(n=11,65 \%)$ and eight (47\%) participants were often not sure of what intervention to trial next. Five $(29 \%)$ participants felt they had a lack of resources and two $(12 \%)$ participants reported that there were competing therapeutic demands such as equipment prescription, functional retraining and discharge planning. Three (18\%) participants reported clinician fatigue as an issue, and three
Table 4 Treatment methods currently used in clinical settings

\begin{tabular}{lll}
\hline Treatment method $^{\mathrm{a}}$ & $\begin{array}{l}\text { Current } \\
\text { use }(\%)\end{array}$ & $\begin{array}{l}\text { Considered } \\
\text { effective (\%) }\end{array}$ \\
\hline Elevation & $17(100)$ & $7(41)$ \\
Compression gloves & $13(76)$ & $4(24)$ \\
Retrograde massage & $13(76)$ & $5(29)$ \\
Compression bandaging & $12(71)$ & $7(41)$ \\
Boxing glove & $9(53)$ & $0(0)$ \\
Manual lymph drainage & $5(29)$ & $2(12)$ \\
Kinesio tape & $3(18)$ & $0(0)$ \\
Range of motion (passive and & $3(18)$ & $4(24)$ \\
active) & & \\
Splinting & $2(12)$ & $0(0)$ \\
Manual Oedema Mobilisation & $2(12)$ & $1(6)$ \\
Functional Electrical & $1(6)$ & $1(6)$ \\
Stimulation & &
\end{tabular}

${ }^{\mathrm{a}}$ Multiple responses permitted

(18\%) participants stated that the patient concordance reduced over time.

\section{Impact of oedema on rehabilitation}

Eleven $(65 \%)$ participants reported that oedema affected ROM and made it 'difficult to exercise', affected body image $(n=5,29 \%)$, caused pain $(n=4,24 \%)$ and stiffness $(n=2,12 \%)$. Furthermore, two participants $(12 \%)$ linked oedema and the 'enlarged limb' to psychosocial impacts with one of these participants linking it to poor hand function and reduced engagement in therapy. Three participants $(18 \%)$ linked oedema to reduced motivation and engagement in therapy. Other comments included the lack of research to provide a basis for any standard of practice for this population. Finally, one participant highlighted that involvement of nursing staff and family support had positive impacts on outcomes.

\section{Discussion}

The literature review highlighted that limited evidence exists regarding oedema in the hands of people with tetraplegia. This study sought to build this knowledge-base, collating information on current clinical practices for oedema management from experts working in predominantly specialised SCI units. The role of occupational therapy for people with tetraplegia includes functional retraining, upper limb management, equipment provision and community reintegration [17]. Oedema management during rehabilitation occurs within the context of these competing therapy goals and experts were seeking guidance to maximise outcomes. 
There was some uncertainty about prevalence and timing of oedema presentation, with participants suggesting that oedema of the hands occurred 'often' whilst reporting an incidence of $23 \%$ of their current patient population. Systematic data collection that follows the recommendations of the ISCIUEBDS [6] would support the development of a clearer understanding of both the prevalence and timing of this issue. It is important for further research to develop a better understanding of causal factors so that recommendations regarding preventive practices can be met. This is particularly important provided the recommendations for early preventative practices [18]. Reports from other clinical areas link oedema to disturbances in the lymphatic system, decreased muscle tone, vascular changes and impaired muscle pump [4, 5, 19, 20]. Participants in this study reported causes linked to biomechanical factors including positioning, lack of movement and disuse of the limb. Multiple factors may contribute to the development and continuing presence of oedema, including the biomechanical factors identified by clinicians as well as underlying physiological processes evident in other diagnostic groups.

Visual inspection to identify the presence of oedema was prevalent despite evidence that interstitial fluid volume can increase by $30 \%$ before oedema is detected by the naked eye [21]. The use of visual inspection may be appropriate in clinical areas where the affected limb can be compared to the unaffected limb. However, relying on visual inspection may not be appropriate for people with tetraplegia who, as suggested in the results, are most likely to develop oedema in both hands. There is a need for an exploration of alternate ways to identify early onset of oedema to inform preventative measures where possible.

The findings highlight that "gold standard" assessment tools, such as the volumetric metre and the tape measure, are not being consistently used. Participants reported that these assessments lacked inter-rater reliability, which contradicts the literature supporting the inter-rater reliability of tape measurement [22]. Further research should explore the reasoning for this belief and address any gaps in knowledge or practice. One consideration may be that the assessments do not determine specific tissue composition changes within a limb [23], an important consideration when working with people with tetraplegia who experience muscle wasting. Finally, ROM has been adopted as an assessment approach that is unique to SCI. Despite established links between oedema and loss of ROM [24], there are no studies that establish ROM as a reliable and valid measure of oedema.

Four treatment methods were regularly applied in clinical practice; elevation, retrograde massage compression gloves and bandaging. This correlates with evidence from the Australasian Lymphology Association stating that use of compression garments is one of the 'cornerstones of lymphoedema' treatments [25]. The boxing glove splint was used by more than half of the participants but not considered effective by any. Cheshire et al. [14] introduced the boxing glove splint to prevent oedema within the first two months of SCI during an era when the newly injured person with tetraplegia was managed with 2-3 months of bed rest. This approach has been retained within clinical practice in Australia despite no evidence for its effectiveness. Medical advancement means that people with tetraplegia are now managed surgically, mobilise earlier, and engage in therapies sooner [26]. It would be appropriate to evaluate the effectiveness of this approach within this new medical context. Finally, the practice evidence gap identified in this study has been reflected in other clinical areas [5, 27], with a particular need to identify treatments with long-lasting effects $[5,13,16]$. The literature highlights the importance of identifying effective treatments to reduce and manage the oedema in the early stages of onset and maintaining the gains long term $[5,28]$.

\section{Limitations}

This study included findings from one small informant group. The inclusion of patients experiencing oedema and other health disciplines providing care may have provided additional information. Participants were not asked to comment on their clinical reasoning and thus we are unable to comment on their reasoning and decision making. Finally, the study did not explore how information about oedema was documented and therefore we are unable to determine whether clinical practice aligned with international recommendations.

\section{Future research}

This survey has highlighted the need for further research to increase understanding of oedema in the hands of people with tetraplegia. Research needs to explore the most accurate assessment tools and effective treatment methods for this population with a focus on clinical utility. A qualitative study with people with tetraplegia who either have experienced or are experiencing oedema in the hands would add depth to our understanding of this issue.

\section{Conclusion}

The clinical experts applied assessment and treatment approaches frequently reported in the general oedema literature but also with some approaches specific to the SCI population. The use of range of motion for assessment and the boxing glove as treatment are unique to SCI clinical practice and both require further investigation. Impacts on 
hand function and engagement in rehabilitation were reported when oedema was present. In addition, there were impacts reported for functional recovery, pain, limitations with ROM and psychological consequences. The effectiveness of current assessment tools and treatment methods requires further research specific to people with tetraplegia with a view to developing clinical practice guidelines.

Acknowledgements We would like to thank and acknowledge all the clinicians who participated in the survey.

\section{Compliance with ethical standards}

Conflict of interest The authors declare that they have no conflict of interest.

Statement of ethics Ethical approval for the study was granted by the Metro South Human Research Ethics Committee and the University of Queensland.

Publisher's note: Springer Nature remains neutral with regard to jurisdictional claims in published maps and institutional affiliations.

\section{References}

1. Anderson KD. Targeting recovery: priorities of the spinal cordinjured population. J Neurotrauma. 2004;21:1371-83.

2. Paralyzed Veterans of America. Early acute management in adults with spinal cord injury. 2008. Available from https://pva. org/research-resources/publications/clinical-practice-guidelines Accessed 30 Jan 2019

3. Palmada M, Shah S, O'Hare K. Issues in the measurement of hand oedema. Physiother Theory Pract. 1998;14:139-48.

4. Artzberger S, White, J. Edema control. In: Gillen G, editor. Stroke rehabilitation: a function-based approach. Missouri: Elsevier Health Services; 2010. p.307-25.

5. Faghri PD. The effects of neuromuscular stimulation-induced muscle contraction versus elevation on hand edema in CVA patients. J Hand Ther. 1997;10:29-34.

6. Biering-Sorensen F, Bryden A, Curt A, Friden J, Harvey LA, Mulcahey MJ, et al. International spinal cord injury upper extremity basic data set (Version 1.1). 2015. Available from https://www.iscos.org.uk/international-sci-upper-extremity-datasets. Accessed 30 Apr 2019.

7. Langbecker D, Hayes SC, Newman B, Janda M. Treatment for upper-limb and lower-limb lymphedema by professionals specializing in lymphedema care. Eur J Cancer Care. 2008;17:557-64.

8. Haesler E. Lymphoedema: Objective assessment using perometry. Wound Pract Res: J Australian Wound Manag Assoc. 2015;23:150-1.

9. Shah CS, Vicini F, Beitsch P, Laidley A, Anglin B, Lyden M. The use of bioimpedance spectroscopy to monitor therapeutic intervention in patients treated for breast cancer-related lymphedema. Int J Radiat Oncol Biol Phys. 2013;46:184-92.

10. Nixon J, Purcell A, Fleming J, McCann A, Porceddu S. Pilot study of an assessment tool for measuring head and neck lymphoedema. Br J Community Nurs. 2014;Suppl:S6,S8-S11.

11. Newman AL, Rosenthall L, Towers A, Hodgson P, Shay CA, Tidhar D. et al. Determining the precision of dual energy x-ray absorptiometry and bioelectric impedance spectroscopy in the assessment of breast cancer-related lymphedema. Lymphat Res Biol. 2013;11:104-9.

12. Fadil A, Aldawsary N, Alshehri MA. A literature review of using compression techniques for the management of lymphoedema. Int J Physiother. 2018;5:23-30.

13. Giang TA, Ong AWG, Krishnamurthy K, Fong KNK. Rehabilitation interventions for poststroke hand oedema: a systematic review. Hong Kong J Occup Ther. 2016;27:7-17.

14. Cheshire DJ, Rowe G. The prevention of deformity in the severely paralysed hand. Spinal Cord.1970;8:48-56.

15. Taradaj J, Halski T, Zdunczyk M, Rajfur J, Pasternok M, Chmielewska D. et al. Evaluation of the effectiveness of kinesio taping application in a patient with secondary lymphedema in breast cancer: a case report. Prz Menopauzalny. 2014;13:73-7.

16. Morris D, Jones D, Ryan H, Ryan CG. The clinical effects of Kinesio(R) Tex taping: a systematic review. Physiother Theory Pract. 2013;29:259-70.

17. Ford S, Keay A, Skipper, D. Occupational therapy intervention for adults with a spinal cord injury: An overview. 2014. Available from: https://www.aci.health.nsw.gov.au/_data/assets/pdf_file/ 0004/155191/occupational-therapy-interventions.pdf Accessed 30 Apr 2019.

18. Sisto S, Druin E, Silwinski M. Management of the Upper Limb in Individuals in tetraplegia. In: Mulcahey MJ, editor. Spinal Cord Injuries: Management and Rehabiliation. Missouri, USA: Elsevier Health Sciences; 2008. p.237-53.

19. Guerts A, Visschers A, Limbeek J, Ribbers G. Systematic review of aetiology and treatment of post stroke hand oedema and shoulder-hand syndrome. Scandanadian J Rehab Med. 2000;32:4-10.

20. Wang JS, Chen SY, Lan C, Wong MK, Lai JS. Neuromuscular electric stimulation enhances endothelial vascular control and hemodynamic function in paretic upper extremities of patients with stroke. Arch Phys Med Rehab. 2004;85:1112-6.

21. Guyton. Capillary fluid exchange, interstitial fluid dynamics and Lymph flow. In: Guyton A, Hall, J, (eds.) Human Physiology and Mechanisms of disease 6th ed. London, UK: Elsevier Health Sciences; 1997. p.130-41.

22. Levenhagen K, Davies C, Perdomo M, Ryans K, Gilchrist L. Diagnosis of Upper Quadrant Lymphedema Secondary to Cancer: Clinical Practice Guideline from the oncology section of the american physical therapy association. Phys Ther. 2017;97:729-45.

23. Czerniec SA, Ward LC, Kilbreath SL. Assessment of breast cancerrelated lymphedema: a comparison of moisture meter and spot bioimpedance measurement. Lymphat Res Biol. 2015;13:10-9.

24. Packer JW, Colditz JC. Bone injuries: treatment and rehabilitation. Hand Clin. 1986;2:81-91.

25. Australasian Lymphology Association. Lymphoedema Management. Available from: http://www.lymphoedema.org.au/index. $\mathrm{cfm} / /$ about-lymphoedema/lymphoedema-management [Accessed 30 January, 2019].

26. Bagnall AM, Jones L, Duffy S, Riemsma RP. Spinal fixation surgery for acute traumatic spinal cord injury. Cochrane Database Syst Rev. 2008;23:CD004725

27. Gustafsson L, Patterson E, Marshall K, Bennett S, Bower K. Efficacy of Compression Gloves in Maintaining Edema Reductions After Application of Compression Bandaging to the StrokeAffected Upper Limb. Am J Occup Ther. 2016;70 (2):7002290030p1-9.

28. Gustafsson L, Walter A, Bower K, Slaughter A, Hoyle M. SingleCase Design Evaluation of Compression Therapy for Edema of the Stroke-Affected Hand. Am J Occup Ther. 2014;68:203-11. 